\title{
Combining Cross-Correlation and Fuzzy Classification to Detect Distributed Denial-of-Service Attacks*
}

\author{
Wei Wei ${ }^{1}$, Yabo Dong ${ }^{1}$, Dongming $\mathrm{Lu}^{1}$, and Guang Jin ${ }^{2}$ \\ ${ }^{1}$ College of Compute Science and Technology, \\ Zhejiang University, Hangzhou 310027, P.R. China \\ ${ }^{2}$ College of Information Science and Engineering, \\ Ningbo University, Ningbo 315211, P.R. China \\ \{weiwei_tc, dongyb, 1dm, d05jinguang\}@zju.edu.cn
}

\begin{abstract}
In legitimate traffic the correlation exists between the outgoing traffic and incoming traffic of a server network because of the request-reply actions in most protocols. When DDoS attacks occur, the attackers send packets with faked source addresses. As a result, the outgoing traffic to the faked addresses does not induce any related incoming traffic. Our main idea is to find changes in the correlation caused by DDoS. We sample network traffics using Extended First Connection Density (EFCD), and express correlation by cross-correlation function. Because network traffic in DDoS-initiating stage is much similar to legitimate traffic, we use fuzzy classification in order to guarantee the accuracy. Experiments show that DDoS traffic can be identified accurately by our algorithm.
\end{abstract}

\section{Introduction}

With the development of the Internet, Distributed Denial of Service (DDoS) becomes a major security threat. In several cases, over ten thousands of attack agents cooperate to send attack packets to flood targets. Attack packets aggregate to block target network, or directly bring target hosts down.

Various methods have been proposed for DDoS detection. J. Mirkovic et al present a detailed conclusion and classification [1] and Q. Li et al give out an analysis on statistical filtering [2]. For statistical detection of DDoS, spectral analysis is adopted. A. Hussain et al use power spectrum to extract fingerprint features from attack stream and DDoS attack scenarios are identified when repeated [3]. Meanwhile, Entropy is used as a summarization tool. A. Lakhina [4] et al get entropy of several packet features and use multi-way subspace method to combine multiple features, then use clustering approach to detect. Besides, L. Feinstein et al give algorithms based on entropy and chi-square [5]. Moreover, many traffic correlation based methods have appeared. S. Jin et al get covariance matrix from several traffic sources and uses the matrix for detection [6]. Wavelet detection methods are also presented. L. Li et al

\footnotetext{
"Funded by National Natural Science Foundation of China (60503061); Zhejiang Provincial Natural Science Foundation (Y104437); Zhejiang Provincial Science and Technology Program (2005C33034); the Program for New Century Excellent Talents in University (No. NCET-04-0535).
} 
compute the difference of wavelet energy function vector between neighborhood traffic windows [7]. Furthermore, Network Traffic's fractal property is used. M. Li uses Long Range Dependent (LRD) traffic pattern recognition for DDoS detection [8]. Y. Xiang et al identify DDoS through detecting self-similarity changes in traffics [9]. In addition, change point methods are widely used. One is R. Blazek et al's sequential change-point and batch sequential detection [10]. And some pattern classification methods are used, such as support vector machine detection in [11].

As to above methods, the main problems are accuracy and performance. Here we propose a DDoS detection method named Cross-Correlation based Fuzzy Classification (CCFC). The method uses Extended First Connection Density (EFCD) to sample outgoing and incoming traffic, and computes the cross-correlation vector sequence. Then fuzzy classification is applied to the sequence for DDoS detection. Experiments show that CCFC is accurate. The rest of this paper is organized as follows: in section 2 we present the principle of CCFC. In section 3 we focus on the implementation of CCFC while some definitions are provided. In section 4, experiment results are shown and explained. Then conclusion is given in section 5 .

\section{The Principle of CCFC}

\subsection{The Definition of EFCD}

EFCD is the count of first connections in a time unit. Here connection is defined with 5 fields in IP header, i.e. source address, source port, destination address, destination port, TCP flags (if have). The first 4 fields are usually used for TCP connection identification. In DDoS attacks, these fields may vary a lot and EFCD value changes abnormally. TCP flags field is used to strengthen the cross-correlation of sampled outgoing and incoming EFCD sequences of legitimate TCP traffic.

Table 1. The EFCD value for ten outgoing TCP packets

\begin{tabular}{lllllll}
\hline Packets & Source IP & Source port & Dest IP & Dest port & TCP flag & Conn order \\
\hline TCP 1 & A & 80 & B & 42957 & 0x26 & 1 \\
TCP 2 & C & 23 & D & 34425 & 0x13 & 2 \\
TCP 3 & A & 80 & B & 42957 & 0x26 & 1 \\
TCP 4 & E & 443 & F & 4256 & 0x13 & 3 \\
TCP 5 & G & 42364 & H & 80 & --- & 4 \\
TCP 6 & A & 80 & B & 42957 & 0x26 & 1 \\
TCP 7 & I & 23467 & J & 23 & 0x36 & 5 \\
TCP 8 & B & --- & A & --- & --- & 6 \\
TCP 9 & B & --- & A & --- & --- & 6 \\
TCP 10 & I & 23467 & J & 23 & 0x36 & 5 \\
\hline
\end{tabular}

Table 1 shows 10 TCP packets in 0.1 seconds. As we can see, they belong to 6 different first connections. So with a time unit of 0.1 seconds, the EFCD is 6 .

$\mathrm{EFCD}$, as a kind of flow-level sample, is more sensitive than pack-level sample in connection based attack. For EFCD, the time unit should be chosen carefully. Bigger 
time unit will shorten the computation time while decrease the detection accuracy. Because RTTs (Round Trip Time) of legitimate network connections are mostly less than 1 second, we choose 0.1 seconds as a time unit to balance the accuracy and performance. Fig. 1 is EFCD with the time unit of 0.1 seconds from MIT Lincoln Laboratory outside data [12].

\subsection{Principle}

The correlation between the traffics of two directions exists because of the requestreply actions in many protocols, such as TCP and many application-level protocols. For example, when a server receives a TCP SYN request, it sends out a TCP ACK packets. And then legitimate client receives the reply and responses data packets with TCP ACK flag set. In above scenario, one EFCD of outgoing traffic contributes to one EFCD of incoming traffic, therefore, there is a positive correlation between values of EFCD of outgoing and incoming traffic and that is shown in Fig. 2.

When DDoS traffic flushes into the server network, most part of EFCD value of incoming attack flows is not caused by EFCD of any earlier outgoing flows. As a result, the correlation between EFCD of the outgoing and incoming traffic is deviated, which can be used for detection. In next section, we will explain our algorithm in details.

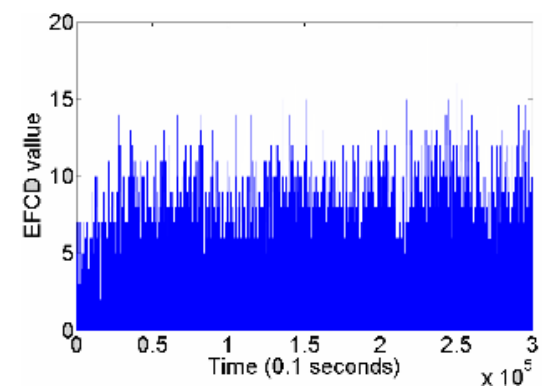

Fig. 1. EFCD sampled values

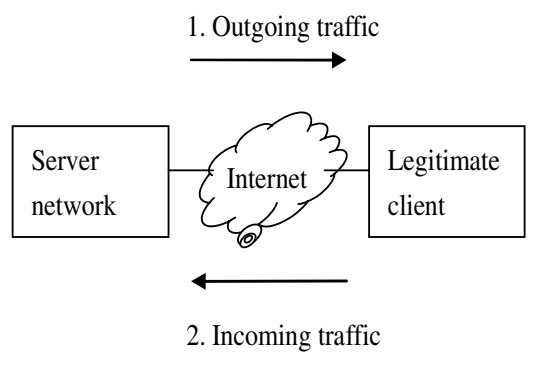

Fig. 2. The request-reply action in normal traffic

\section{The Algorithm of CCFC}

\subsection{Computing Cross-Correlation Vector Sequence}

Correlation has been used in network anomaly detection, such as in [6]. It can be well described by cross-correlation function, which is definded as below [13]:

$$
\begin{aligned}
& \mathrm{R}_{\mathrm{Xy}}(t, t+\tau)=\frac{\operatorname{cov}(X(t), Y(t+\tau))}{\sigma_{x(t)^{\sigma} y(t+\tau)}^{\sigma}} \\
& =\frac{\mathrm{E}((X(t)-\mu(X(t)))(Y(t+\tau)-\mu(Y(t+\tau))))}{\sqrt{\left(\mathrm{E}\left(X^{2}(t)\right)-(\mathrm{E}(X(t)))^{2}\right)} \sqrt{\left(\mathrm{E}\left(Y^{2}(t+\tau)\right)-(\mathrm{E}(Y(t+\tau)))^{2}\right)}} .
\end{aligned}
$$


Here $X$ and $Y$ are two random processes and $\mathrm{R}_{\mathrm{xy}}(t, t+\tau) \in[-1,1]$ is their crosscorrelation function. $\tau$ is the correlation step (usually referred to as 'lags') which is an integer. $X(t)$ represents the random variable of $X$ at time $t$ while $Y(t+\tau)$ represents the random variable of $Y$ at time $t+\tau, t \in[0,+\infty) . \mu(X(t))$ and $\mu(Y(t+\tau))$ are mathematical expectations of $X(t)$ and $Y(t+\tau)$ respectively. $\sigma_{X(t)}$ and $\sigma_{Y(t)}$ are standard deviations of $X(t)$ and $Y(t+\tau)$. For given $\mathrm{t}, \mathrm{R}_{\mathrm{xy}}(t, t+\tau)$ can be written as $\mathrm{R}_{\mathrm{xy}}(\tau)$. Note that $\mathrm{R}_{\mathrm{xy}}(\tau)=\mathrm{R}_{\mathrm{yx}}(-\tau)$.

Because in legitimate traffic the RTT is mostly less than 0.5 seconds, we sample traffic using time unit of 0.1 second. As a result, we compute 1 to 5 step crosscorrelation values which are sufficient to represent the cross-correlation of legitimate traffic. For two sequences, their cross-correlation values with different $\tau$ compose cross-correlation vector. Here we set $\tau \in\{1,2,3,4,5\}$, the cross-correlation vector of $X$ and $Y$ is $\left\{\mathrm{R}_{\mathrm{xy}}(1), \mathrm{R}_{\mathrm{xy}}(2), \mathrm{R}_{\mathrm{xy}}(3), \mathrm{R}_{\mathrm{xy}}(4), \mathrm{R}_{\mathrm{xy}}(5)\right\}$.

In this step we will divide EFCD sequence into several subsequences according to a configured time window and compute the cross-correlation between the outgoing traffic and incoming traffic in a same time window. Here a time window of 3 seconds is suitable for accurate computation and detection, i.e. there are $30 \mathrm{EFCD}$ values in a subsequence.

The 1 to 10 step cross-correlation vector of legitimate and DDoS traffic are shown in Fig. 3. The correlation step and time window's width should be chosen carefully. The sampling with big window needs less computation, but it is less accurate than with small window. The time unit should be chosen carefully. Bigger time unit will shorten the computation time while decrease the detection accuracy. A similar balance should also be considered for the choosing of correlation step.

\subsection{Defining Membership Functions}

Because the cross-correlation differences between legitimate and DDoS traffic are not always obvious, the fuzzy classification can be a good way to differentiate them.
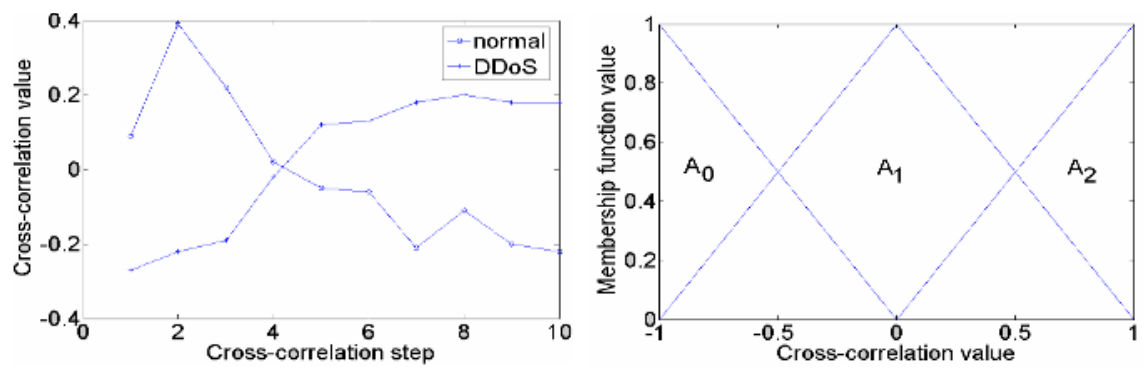

Fig. 3. Typical legitimate and ddos cross-Fig. 4. Fuzzy sets for every axis of pattern correlation sequence (step 1 to 10 ) space

The sequence of cross-correlation vector is used as the input for fuzzy classification module. Cross-correlation vector is used as the pattern and sequence of cross-correlation vector as the pattern subspace. Dimension number is the length of cross-correlation vector. $K$ fuzzy sets exist in each dimension of the pattern space and 
here we choose $K=3$. That is enough for our fuzzy classification. We partition every axis of the pattern space into 3 fuzzy sets $A_{1}, A_{2}, A_{3}$.

Like usual operation, symmetric triangular membership functions are used and the corresponding result is shown in Fig. 4.

\subsection{Training Fuzzy Classifier Using History Data}

The input pattern is used as input variables and the output is mapped to result classes. Here the main problem is how to generate fuzzy 'If-Then' rules from history data. In this paper, we use an improved classification method in [14]. Following the definition in (1), the training pattern space is 5-dimension and can be expressed as:

$$
[-1,1] \times[-1,1] \times[-1,1] \times[-1,1] \times[-1,1] \text {. }
$$

History data are $N$ patterns, denoted as $V_{m}=\left\{\left(v_{m, 1}, \quad v_{m, 2}, \quad v_{m, 3}, \quad v_{m, 4}\right.\right.$, $\left.\left.v_{m, 5}\right) \mid m=1,2,3 \ldots, N\right\}$, which belong to $M$ output classes, here $M=2$, i.e. $N$ patterns belong to either legitimate or DDoS class.

The label of rules is shown in Fig 5. ' $A_{x}$ ' represents fuzzy set for one dimension, for each dimension there are three fuzzy sets $A_{0}, A_{1}, A_{2}$. We just draw the first 2 dimensions here. ' $R_{i j . . .}$ ' represents fuzzy rule, and its subscript is a 5-character string written as an array $S$, where $S(\cdot)$ can be ' 0 ', ' 1 ' and ' 2 '. For one pattern $V_{m}$, a rule $R_{S}$ can be described as bellows:

If $v_{m, 1}$ is $A_{S(1)}, v_{m, 2}$ is $A_{S(2)}, v_{m, 3}$ is $A_{S(3)}, v_{m, 4}$ is $A_{S(4)}$ and $v_{m, 5}$ is $A_{S(5)}$, then $V_{m}$ belongs to output class $n_{S}\left(n_{S} \in\{1,2\}\right)$ with Certainty Factor $C F_{S}$, which is the possibility of this rule belonging to the output class.

For each rule, its output class $n_{S}$ and $C F_{S}$ are decided in training step. The generations of 'If-Then' rules are given below. Firstly, for each rule $R_{S}$, the probability for decision $P_{n, S}$ is computed as follows:

$$
P_{n, S}=\sum_{V_{m} \in \operatorname{class}} \prod_{n}^{L} \rho_{S(t)}\left(v_{m, t}\right)
$$

In (3), class $n$ is one of the classes the patterns finally belong to. Here $n \in\{1,2\}$. $L$ is the dimension number of input patterns and $L=5 . v_{m, t}$ is the value in dimension $t$ of pattern $V_{m} . \rho_{S(t)}$ is the fuzzy set $\mathrm{A}_{S(t)}$ 's membership function and $S(t)$ is the value in index $t$ of vector $S$. For rule $R_{S}$, we can get $P_{1, S}$ and $P_{2, S}$. If $P_{1, S}>P_{2, S}$, then the output class $n$ is class 1 , else if $P_{1, S}<P_{2, S}$, then the output class $n$ is class 2 . If $P_{1, S}=P_{2, S}$, the output class is undecided, so when implementation, this rule is classified into one class with $C F_{S}=0$. At last the $C F_{S}$ of this rule is computed as follows, where the rule belongs to class $t^{\prime}$.

$$
C F_{S}=\left(P_{t^{\prime}, S}-\left(\sum_{t=1, t \neq t^{\prime}}^{M} P_{t, S}\right) /(M-1)\right) / \sum_{t=1}^{M} P_{t, S} .
$$

\subsection{Using Fuzzy Classifier to Detect}

In this step we use the fuzzy classifier trained in 3.3 to classify unknown patterns. For one input pattern $V_{m}$, we compute $q_{\max }$ : 


$$
q_{\text {max }}=\max \left\{\left(\prod_{t=1}^{L} \rho_{S(t)}\left(v_{m, t}\right)\right) \bullet C F_{S} \mid S \in \text { set of all rules }\right\} .
$$

With the $q_{\max }$ and the corresponding rule $R_{S}$, the pattern $V_{m}$ is assigned to the class $n_{S}$ that the rule $R_{S}$ belongs to. If $q_{\max }$ is can not be decided and the corresponding rules don't belong to a same output class, the classifier doesn't give a result. And if the result is DDoS class then an alarm is generated.

\section{Experiment Results}

We used MIT Lincoln Laboratory's data in [12] to train and evaluate our algorithm. The attack events are shown in Fig 6, where x-axis is the time windows sequence number, and in $\mathrm{y}$-axis 0 stands for legitimate while 1 stands for DDoS attack. The DDoS attacks occur at time window 5 to 7 and 19 to 21 .

The detection results are shown in Fig. 7. But for the result, there are false positive alarms in time window 11 and 15. Through observing original sampling data, we found at time window 11 and 15 the percentage of TCP traffic is unusually low and UDP traffic has a burst. And in the situation most of outgoing packets are UDP packets and legitimate clients do not reply to what they received, as a result, the correlation is weak.

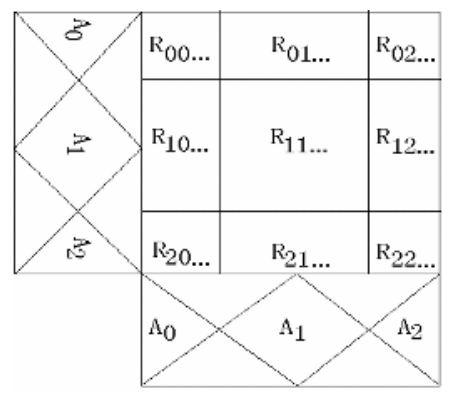

Fig. 5. The label of rules in the 1 st and 2 nd dimension of 5 dimensions

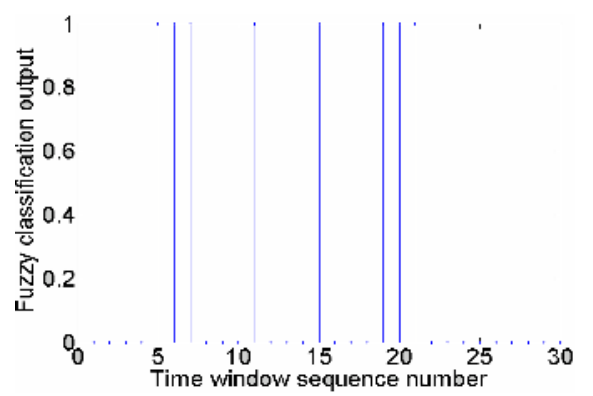

Fig. 7. Detection result

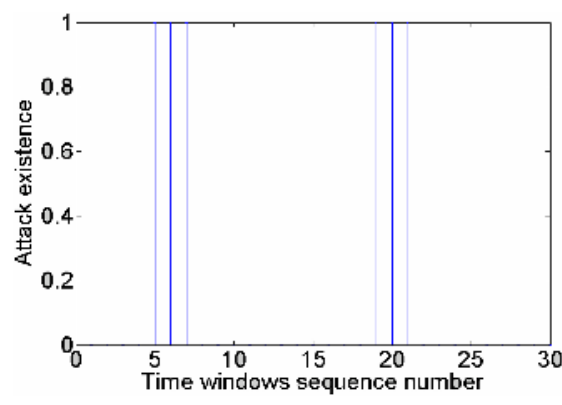

Fig. 6. Attack existence

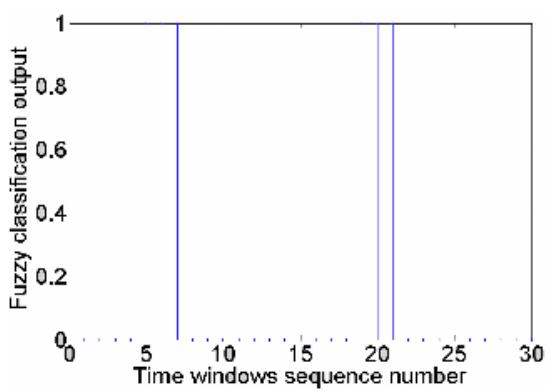

Fig. 8. Detection result after filtering 
This type of false positive alarms can be erased through filtering. The main principle is that neighborhood alarms strengthen each other, i.e. alarms are clustered and given a trust value. If the value is greater than a given value, $T r$, alarms in this cluster are reported. When an alarm rises, if there exists a cluster, the trust value is updated; otherwise, a new cluster is created with an initial trust value. When alarm stops for several time windows, $T c$, the existing cluster is removed.

The detection result after filtering is accurate as shown in Fig. 8. We evaluate the algorithm with several other data sets [12] and get similar results.

\section{Conclusion}

In this paper we proposed a new fuzzy classification algorithm for DDoS detection. The algorithm is based on the fact that DDoS attacks can deviate the cross-correlation of EFCD of network traffic.

In this algorithm, we get EFCD sequence of outgoing and incoming traffic of server network and then transform it into 5-step cross-correlation sequences. Then we use legitimate and DDoS data to train fuzzy classification and used it to detect DDoS attacks. The experiment results show that CCFC is accurate enough.

Higher dimension in fuzzy classification could increase the accuracy of detection, while increase the computation consumption. We plan to improve this algorithm with higher dimension pattern space for application in real time.

\section{References}

1. Mirkovic, J., Reiher, P.: A taxonomy of DDoS attack and DDoS defense mechanisms. Computer Communication Review, Vol. 34(2), 39-53, 2004.

2. Li, Q., Chang, E. C., Chan, M. C.: On the effectiveness of DDoS attacks on statistical filtering. In Proceedings of IEEE INFOCOM 2005, March 2005.

3. Hussain, A., Heidemann, J., Papadopoulos, C.: Identification of Repeated Denial of Service Attacks. In Proceedings of IEEE INFOCOM 2006, April 2006.

4. Lakhina, A., Crovella, M., Diot, C.: Mining Anomalies Using Traffic Feature Distributions. In Proceedings of ACM SIGCOMM 2005, August 2005.

5. Laura, F. Dan, S. Statistical Approaches to DDoS Attack Detection and Response. In Proceedings of DARPA Information Survivability Conference and Exposition, Vol. 1, 303-314, 2003.

6. Jin, S., Yeung, Y. D.: A covariance analysis model for DDoS attack detection. In Proceedings of IEEE International Conference on Communications, Vol. 4, 1882-1886, 2004.

7. Li, L., Lee, G.: DDoS attack detection and wavelets. Computer Computer Communications and Networks, 421-427, 2003.

8. Li, M.: An approach to reliably identifying signs of DDoS flood attacks based on LRD traffic pattern recognition. Computers and Security, Vol. 23(7), 549-558, 2004.

9. Xiang, Y., Lin, Y., Lei, W. L., Huang, S. J.: Detecting DDoS attack based on network selfsimilarity. IEEE Proceedings Communications, Vol. 151(3), 292-295, 2004.

10. Blazek, R., Kim, H., Rozovskii, B., Alexander, T.: A novel approach to detection of "denial-of-service" attacks via adaptive sequential and batch-sequential change-point detection methods. In Proceedings of the 2001 IEEE Workshop on Information Assurance and Security United States Military Academy, 2001. 
11. Shon, T., Kim, Y., Lee, C.: Jongsub Moon: A machine learning framework for network anomaly detection using SVM and GA. In Proceedings of Systems, Man and Cybernetics (SMC) Information Assurance Workshop, 176-183, 2005

12. http://www.1l.mit.edu/IST/ideval/data/2000/LLS_DDOS_1.0.html

13. Box, G.E.P., Jenkins, G.M., Reinsel, G.C.: Time Series Analysis: Forecasting and Control, Third edition, Prentice Hall, 1994.

14. Ravi, V., Zimmermann, H. J.: Fuzzy rule based classification with FeatureSelector and modified threshold accepting. European Journal of Operational Research, Vol. 123(1), 1628,2000 . 